ARTÍCULO

\title{
Caracterización del sustrato bentónico del arrecife Tuxpan, Veracruz, México, con énfasis en la comunidad de corales escleractinios
}

\author{
Characterization of benthic substrate of Tuxpan reef, Veracruz, Mexico with emphasis on \\ scleractinian coral community
}

\section{Carlos González-Gándara ${ }^{* *}$ y José de Jesús Salas-Pérez ${ }^{\text {** }}$}

\author{
${ }^{1}$ Laboratorio de Arrecifes Coralinos, Facultad de Ciencias Biológicas y Agropecuarias, Universidad Veracruzana, Carr. Tuxpan-Tampico \\ km 7.5, Col. Universitaria, CP 92860, Tuxpan, Veracruz, México. **jsalasp39@yahoo.es \\ *Autor corresponsal: cggandara@hotmail.com
}

\begin{abstract}
Benthic substrate of Tuxpan reef, Veracruz, Mexico, with emphasis on scleractinian coral community was characterized in order to define a model of richness and coral cover for platform reefs of Veracruz. 117 phototransects were distributed in 4 zones and 16 reef habitats during 2015. A total of 2,920 photographs were taken on transects with $25 \mathrm{~m}$ in length for estimate morpho functional groups (MFG) and coral cover using the Coral Point Count program. Richness and coral cover were compared in order to determine their similarity between zones and reef habitats regarding MFG and coral cover using statistical or ecological tests. Scleractinian coral community of Tuxpan reef was composed by 36 species and the richness showed differences between zones and was greater in reef slopes. According with non-metric multidimensional scaling (NMDS) and ANOSIM, the GMF and species coral cover characterized 4 reef zones: reef flat, reef crest, windward slope and leeward slope. Leeward coral cover was inversely proportional to depth, higher values were observed on 3 to $15 \mathrm{~m}$ depth. Windward coral cover did not show relationship with depth, the higher values were detected on 10 to $20 \mathrm{~m}$. The characterization of Tuxpan reef could serve as model of platform reefs of Veracruz as well as to management plans of this Natural Protected Area, especially on snorkel and diving practices.
\end{abstract}

Key words: Characterization, coral cover, morpho functional groups, Gulf of Mexico

Resumen.- Se caracteriza el sustrato bentónico del arrecife Tuxpan, Veracruz, México con énfasis en la comunidad de corales escleractinios para definir el patrón de riqueza y cobertura coralina de las formaciones tipo plataforma de Veracruz.117 fototransectos, fueron distribuidos en 4 zonas y 16 ambientes arrecifales durante 2015. Se tomaron un total de 2.920 fotografías sobre transectos de $25 \mathrm{~m}$ para estimar la cobertura con el programa Coral Point Count. La riqueza y cobertura coralina fueron comparadas para determinar su semejanza entre zonas y ambientes arrecifales, a partir de los grupos morfo funcionales (GMF), usando pruebas estadísticas o ecológicas. 36 especies componen la comunidad de corales escleractinios y la riqueza coralina mostró diferencias entre zonas, siendo mayor en las pendientes. De acuerdo con el análisis de escalamiento multidimensional no métrico (NMDS) y ANOSIM, los GMF y la cobertura específica de corales caracterizaron 4 zonas arrecifales: planicie, cresta, pendiente de barlovento y pendiente de sotavento. La cobertura coralina en sotavento, es inversamente proporcional a la profundidad, donde los valores más altos fueron observados entre 3 y $15 \mathrm{~m}$. En barlovento, no se evidenció una relación de la cobertura coralina con la profundidad y los valores más altos se registraron entre 10 y $20 \mathrm{~m}$. La caracterización de las zonas del arrecife Tuxpan puede servir como modelo de los arrecifes tipo plataforma de Veracruz así como para los planes de manejo de esta Área Natural Protegida, especialmente en las actividades de esnórquel y buceo.

Palabras clave: Caracterización, cobertura coralina, grupos morfo funcionales, Golfo de México

\section{INTRODUCCIÓN}

Los arrecifes coralinos del suroeste del Golfo de México se agrupan en 3 áreas: el Sistema Arrecifal Lobos Tuxpan (SALT), El Sistema Arrecifal Veracruzano (SAV) y el Sistema Arrecifal Los Tuxtlas (SAT) (Ortiz-Lozano et al. 2013) e incluyen arrecifes de tipo plataforma, marginales y bancos sumergidos. La mayoría de estos sistemas se desarrollan bajo condiciones de turbidez y estrés térmico (Tunnell 1988, 1992) debido a su cercanía a la costa (HortaPuga et al.2015) y al drenaje de las 10 cuencas hidrológicas que ocurren en el estado de Veracruz (Pérez-Maqueo et al. 2011). La geomorfología de los arrecifes tipo plataforma incluye 4 zonas: cresta arrecifal, laguna, pendiente de barlovento y pendiente de sotavento, las cuales presentan diferencias en su desarrollo y componentes bentónicos (Lara et al. 1992, Jordan-Dahlgren \& Rodríguez-Martínez 2003, Chávez et al. 2010, Horta-Puga et al. 2015) que resultan de la exposición al oleaje, las corrientes marinas (Rogers et al. 2016), los sedimentos suspendidos y la turbidez (Erftemeijer et al. 2012, Fabricious et al. 2014, Wenger et al. 2015). 
Las investigaciones efectuadas en los arrecifes plataforma de Veracruz se han centrado en la composición y cobertura coralina de las pendientes, considerando profundidades menores a $20 \mathrm{~m}$. Sólo en algunos arrecifes (Blanquilla, Cabezo), se tiene información de la comunidad coralina de la planicie arrecifal (Horta-Puga et al. 2015), por lo que el conocimiento de la cobertura coralina a escala de zonas y hábitats arrecifales es limitado. Por otra parte, la cobertura de corales registrada en las pendientes de los arrecifes de Veracruz, es contrastante, ya que en algunos casos (Anegada de Afuera, Cabezo, En Medio, Lobos), es mayor en sotavento y en otros (Blanquilla, Sacrificios y Pájaros), es más alta en barlovento (Lara et al. 1992, Escobar-Vázquez \& Chávez 2012, Horta-Puga et al. 2015).

Dado que las formaciones arrecifales de Veracruz son áreas naturales protegidas, es necesario conocer a detalle al menos la riqueza y cobertura coralina, particularmente cuando se permiten actividades recreativas como el esnórquel y el buceo. Estas actividades producen un efecto sobre las comunidades coralinas, el cual varía en función de la profundidad, la carga de buceo y de la experiencia de los buzos (Muthiga \& McClanahan 1997, Castro-Triana \& Pereira-Cháves 2016) así como de la composición y cobertura bentónica del arrecife o de la morfología de las colonias (Hawkins \& Roberts 1992).

En el norte de Veracruz, el arrecife Tuxpan forma parte del Área de Protección de Flora y Fauna Sistema Arrecifal Lobos Tuxpan (CONANP 2014) y es uno de los más visitados por turistas. Las comunidades bentónicas de este sistema han sido descritas parcialmente por Martos (1993) y Alfaro (2012), indicando los contrastes en la riqueza y distribución de corales en las pendientes, así como una caracterización general del fondo. Para favorecer la preservación del arrecife Tuxpan, es necesario que se conozcan los atributos bentónicos, especialmente la riqueza y cobertura coralina. Por lo anterior, en la presente investigación se evaluó la riqueza, la cobertura de los grupos morfo funcionales y de corales en el arrecife Tuxpan relacionándolos con las zonas arrecifales y la profundidad. Esto permitirá conocer los patrones de riqueza y cobertura coralina por zonas en arrecifes tipo plataforma veracruzanos y podrán ser utilizados para fundamentar las estrategias de conservación del sistema.

\section{Materiales Y MÉTODOS}

\section{ÁREA DE ESTUdio}

El arrecife Tuxpan se localiza en el Golfo de México, frente a las costas de Tuxpan, Veracruz y entre los puntos geográficos $21^{\circ} 01$ '38”N y $97^{\circ} 11^{\prime} 40^{\prime}$ W (Fig. 1). Es una formación ovalada de tipo plataforma con su eje mayor orientado en sentido sureste noreste, que oscila entre los 0,5 y $27 \mathrm{~m}$ de profundidad. Este sistema presenta 4 zonas arrecifales: una pendiente abrupta en sotavento, caracterizada por una cobertura coralina mayor al 20\% (Martos 1993) y cuya profundidad va de 3 a $27 \mathrm{~m}$; una pendiente en barlovento de perfil suave, expuesta al oleaje, con una profundidad de 3 a $27 \mathrm{~m}$; la planicie arrecifal que contiene dos hábitats definidos: pastos marinos y restos de coral muerto, cuya profundidad varía entre 0,5 y $1,5 \mathrm{~m}$; y la cresta arrecifal que delimita las pendientes, expuesta al oleaje y posee dos hábitats: corales masivos con Halimeda y Roca con algas costrosas, Acropora y Millepora (Alfaro 2012).

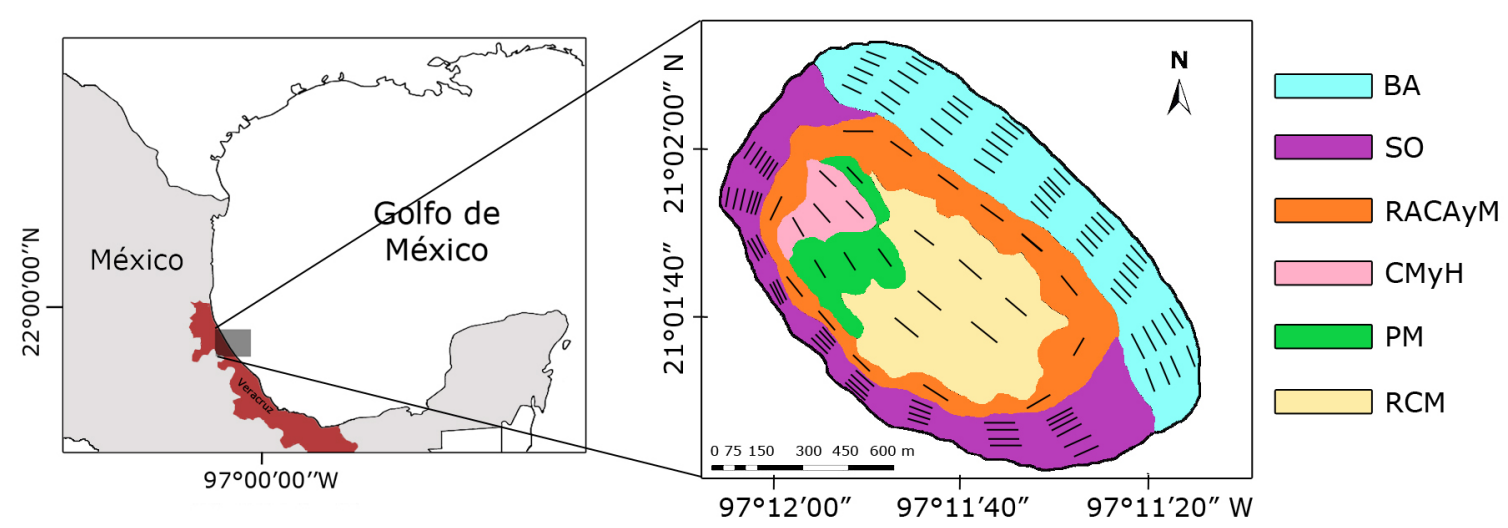

Figura 1. Localización geográfica del arrecife Tuxpan, Veracruz, México. $B A=$ pendiente de barlovento, SO= pendiente de sotavento, RACAyM= roca con algas costrosas, Acropora y Millepora, $\mathrm{CMyH}=$ corales masivos y Halimeda, $\mathrm{PM}=$ pastos marinos, $\mathrm{RCM}=$ restos de coral muerto. Las líneas negras corresponden a los sitios de muestreo / Geographic location of Tuxpan Reef, Veracruz, Mexico. BA= windward slope, SO= leeward slope, RACAyM= rock with encrusting coralline algae, Acropora and Millepora, $\mathrm{CMyH}=$ massive corals and $\mathrm{Halimeda}, \mathrm{PM}=\mathrm{seagrasses}, \mathrm{RCM}=$ fragments of dead coral. Black lines correspond to sampling sites 
Se realizaron 117 fototransectos durante 2015, mediante buceo libre y con equipo autónomo SCUBA. El número y distribución de los transectos (48 en la pendiente de barlovento, 44 en la pendiente de sotavento, 10 en la planicie y 17 en la cresta arrecifal) se determinó a partir de la proporción que representa cada zona y hábitat arrecifal (Fig. 1) y de la variación en la cobertura coralina en cada una de ellas. Durante las inmersiones, se identificaron las especies de corales escleractinios, milleporinos y estilasterinos in situ, considerando los atributos morfológicos que los distinguen de acuerdo a la guía de Humann \& Deloach (2013). Cuando no fue posible identificar los corales en el campo, se tomaron muestras para determinarlos en el laboratorio, con base en las publicaciones de: Veron (2000), Budd et al. (2012) y Zlatarsky \& Martínez-Estalella (2018). La lista fue completada con los registros existentes en la bibliografía publicada previamente y los nombres científicos fueron corroborados en la página web de WoRMS (2019). Para determinar la suficiencia del muestreo se estimó la riqueza a partir de los modelos no paramétricos de: Chao2, Jack 1, Jack 2 y Bootstrap con el programa EstimateS versión 9.1.0 (Colwell 2013).

Para evaluar la cobertura con relación a la profundidad y los ambientes arrecifales, se establecieron rangos artificiales, basados en los estratos utilizados en las evaluaciones rápidas de arrecifes coralinos (e.g., AGRRA, ReefCheck) y en las capas de profundidad utilizadas en las investigaciones de arrecifes veracruzanos (Lara et al. 1992, Horta-Puga et al. 2015), las cuales fueron: $3-5 ; 5-10 ; 10-15 ; 15-20 ; 20-25$ y $>25 \mathrm{~m}$. Asimismo, se determinaron los ambientes más conspicuos de la cresta (corales masivos con Halimeda y roca con algas costrosas, Acropora y Millepora) y la planicie arrecifal (pastos marinos y restos de coral muerto). En cada sitio de muestreo, dependiendo del área ocupada por el estrato o hábitat, se tomaron entre 3 y 12 fototransectos, utilizando una línea de $25 \mathrm{~m}$ de longitud, ubicada paralelamente al eje principal del sistema, para lograr uniformidad en la profundidad. Se tomaron 2.920 fotografías con una cámara fotográfica Cannon Shot de $12 \mathrm{mgp}$ montada sobre un bastón y colocada a $1 \mathrm{~m}$ de la superficie en las pendientes y a $0,5 \mathrm{~m}$ en la planicie y cresta arrecifal debido a la escasa profundidad de estas zonas. Posteriormente, se determinaron los grupos morfo funcionales, a partir de los criterios de la metodología AGRRA (Lang et al. 2015) y las especies de coral, analizando las fotografías con el programa Coral Point Count with Excel extensions V4.1, considerando 25 puntos al azar (Kohler \& Gill 2006).

Para detectar la semejanza entre zonas o ambientes arrecifales, se realizó un análisis de escalamiento multidimensional no métrico (NMDS) con distancia de Bray-Curtis (Clarke 1993) a partir de la cobertura de los grupos morfo funcionales y de corales utilizando el programa PAST versión 3.25 (Hammer et al. 2001). Para probar sí las agrupaciones establecidas a través del NMDS difieren significativamente entre ellas, se aplicó un análisis de similitud (ANOSIM) de una vía, utilizando a los hábitats como factor de clasificación con el programa PRIMER-E v7 (Clarke \& Gorley 2006). Las diferencias en la cobertura coralina fueron valoradas con la prueba de Kruskal-Wallis o mediante un ANOVA, en función de que los datos cumplieran o no, los supuestos (homogeneidad de varianza y distribución normal). Para el análisis posterior, se utilizó la prueba t-Student o la prueba $U$ de Mann-Whitney. Finalmente, se relacionó la cobertura coralina con la profundidad en las pendientes mediante una correlación. Para los análisis estadísticos de utilizó el programa Statgraphics Plus 5.1 (Statistical Graphics 2001) ${ }^{1}$.

\section{Resultados}

El sustrato bentónico del arrecife Tuxpan estuvo conformado por 7 grupos morfo funcionales: coral, invertebrados sésiles, macroalgas, roca cubierta de algas costrosas, roca cubierta de algas césped, pastos marinos y material inerte (roca, restos de coral, arena). Las diferencias en las coberturas de los diferentes grupos morfo funcionales permitió caracterizar las zonas y los hábitats arrecifales de la formación Tuxpan. Por ejemplo, los corales cubren una alta proporción en la pendiente de sotavento $(30,2 \%$ en promedio) que contrasta con el $8,3 \%$ en promedio de la pendiente de barlovento; las algas costrosas fueron más abundantes en las áreas de la pendiente de barlovento ( $31,5 \%$ en promedio) y en la cresta (19,5\% en promedio); y las algas césped mostraron altos porcentajes en las capas de más de $15 \mathrm{~m}$ en sotavento (36,4\% en promedio) y de 3 a $10 \mathrm{~m}$ en barlovento $(43,1 \%$ en promedio) (Fig. 2).

${ }^{1}$ Statistical Graphics. 2001. Statgraphics Plus for Windows. Version 5.1. Statgraphics Technologies, Inc. <http://www.statgraphics.com> 


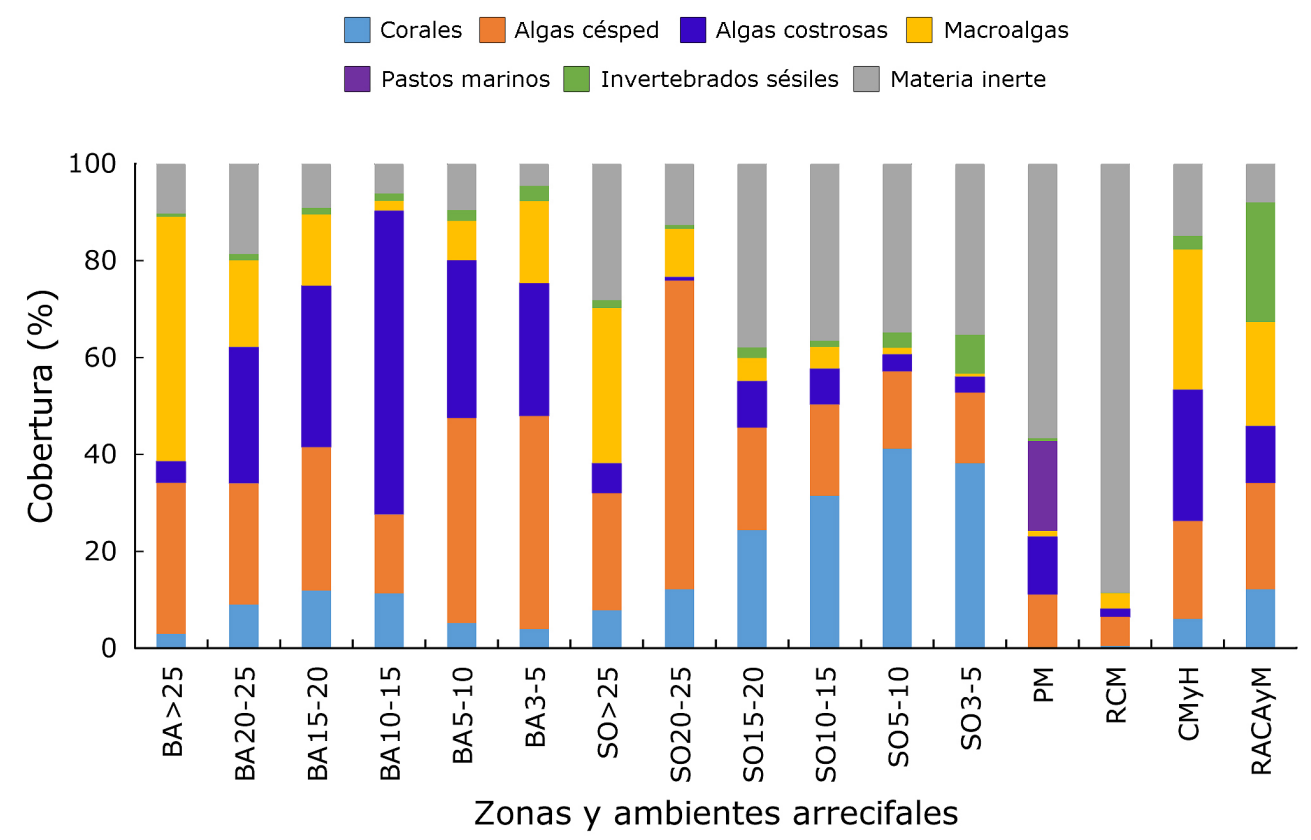

Figura 2. Porcentaje de cobertura de los grupos morfo funcionales del arrecife Tuxpan, Veracruz, México. BA= pendiente de barlovento; $\mathrm{SO}=$ pendiente de sotavento, RACAyM= roca con algas costrosas, Acropora y Millepora, $\mathrm{CMyH}=$ corales masivos y $\mathrm{Halimeda}$, $\mathrm{PM}=$ pastos marinos, $\mathrm{RCM}=$ restos de coral muerto. 3-5, 5-10, 10-15, 15-20, 20-25 y >25 corresponden a los ámbitos de profundidad / Percent cover of morpho functional groups at Tuxpan reef, Veracruz, Mexico. BA= windward slope; SO= leeward slope, RACAyM= rock with encrusting coralline algae, Acropora and Millepora, $\mathrm{CMyH}=$ massive corals and Halimeda, $\mathrm{PM}=$ seagrasses, RCM= fragments of dead coral. 3-5, 5-10, 10-15, 15-20, 20-25 and >25 correspond to depth layers

Los géneros de algas costrosas más conspicuos del arrecife Tuxpan fueron: Lithophyllum, Neogoliton y Porolithon cuyos valores más altos se observaron en la pendiente de barlovento y en la cresta arrecifal. De especial interés fueron los valores estimados en las capas de 5 a $20 \mathrm{~m}$, que oscilaron entre 32,6 y $62,8 \%$ (Fig. 2). Las macroalgas estuvieron representadas por 6 géneros: Dyctiota y Laurencia en la pendiente de barlovento, especialmente en las capas mayores a $15 \mathrm{~m}$ de profundidad; Galaxaura y Amphiroa en las partes someras (3-10 m) de esta pendiente; y Halimeda y Caulerpa en la cresta y planicie arrecifal. En la pendiente de sotavento, este grupo morfo funcional presentó una tendencia a incrementar su porcentaje con la profundidad. Por su parte, las algas césped, mostraron una proporción ligeramente mayor en barlovento $(31,4 \pm 4,3 \%)$ con respecto a la pendiente de sotavento (26,4 $\pm 7,6 \%)$ (Fig. 2).

Los invertebrados sésiles estuvieron representados por el gorgonáceo Erythropodium caribaeorum y esponjas de los géneros: Cliona, Amphimedon, Ircinia y Aplysina. Los valores más altos fueron estimados en la cresta arrecifal (13,7\% en promedio) y en la capa de 3 a $5 \mathrm{~m}$ de la pendiente de sotavento $(8,0 \%$ en promedio $)$, debido principalmente al crecimiento de E. caribaeorum sobre las colonias de Orbicella annularis. Finalmente, el material inerte conformado por: arena, restos de coral muerto y roca coralina fue más notable en la laguna arrecifal
$(72,6 \pm 16,0 \%)$, aunque en la pendiente de sotavento fue importante la presencia de arena entre las formaciones coralinas (30,9 $\pm 3,9 \%)$ (Fig. 2).

La comunidad de corales escleractinios del arrecife Tuxpan estuvo compuesta por 36 especies, 19 géneros y 14 familias, además de los hidrocorales Millepora alcicornis y Stylaster roseus (Tabla 1). De acuerdo con los estimadores no paramétricos (Chao2, Jack1, Jack2 y Bootstrap) la riqueza coralina en este sistema puede ser de hasta 41 especies (Fig. 3). Al comparar las observaciones de las especies por zonas, incluidas los registros previos al presente estudio, se registraron 28 especies en barlovento, 30 en sotavento, 6 en la laguna y 13 en la cresta (Tabla 1). De acuerdo a la prueba de Kruskal-Wallis $\left(\mathrm{H}_{(3,113)}=46,25\right.$, $P<0,05)$, hay diferencias significativas en la riqueza coralina en función de las zonas, con valores más altos en las pendientes y menores en la laguna arrecifal.

La cobertura general promedio de los corales en el arrecife Tuxpan fue de 15,8\% y varió en función de las zonas arrecifales, mostrando los valores promedio más altos, en la pendiente de sotavento $(30,2 \pm 1,9 \%)$, seguido de la pendiente de barlovento $(8,3 \pm 1,1 \%)$, la cresta $(7,8 \pm 1,6 \%)$ y la laguna arrecifal $(0,4 \pm 0,2 \%)$ (Fig. 4). La prueba de Kruskal-Wallis $\left(\mathrm{H}_{(3,113)}=77,33, P<0,05\right)$ indicó que existen diferencias significativas entre las zonas. La prueba posterior demuestra que la cobertura 
Tabla 1. Lista de corales escleractinios, milleporinos y estilasterinos registrados en las zonas del arrecife Tuxpan, Veracruz, México / Checklist of scleractinian, milleporids and stylasterins recorded by zone at Tuxpan reef, Veracruz, Mexico

\begin{tabular}{|c|c|c|c|c|c|}
\hline \multirow{2}{*}{ Familia } & \multirow{2}{*}{ Especie } & \multicolumn{4}{|c|}{ Zonas arrecifales } \\
\hline & & Barlovento & Sotavento & Planicie & Cresta \\
\hline Milleporidae & Millepora alcicornis Linnaeus, 1758 & 1 & 1 & & 1 \\
\hline Stylasterinidae & Stylaster roseus (Pallas, 1766) & 1 & 1 & & \\
\hline Astroceniidae & Stephanocoenia intersepta (Lamarck, 1816) & 1 & 1 & & \\
\hline \multirow[t]{3}{*}{ Pocilloporidae } & Madracis auretenra Locke, Weil \& Coates, 2007 & & 1 & & \\
\hline & Madracis decactis (Lyman, 1859) & 1 & 1 & & \\
\hline & Madracis pharensis pharensis (Heller, 1868) & 1 & & & \\
\hline \multirow[t]{2}{*}{ Acroporidae } & Acropora cervicornis (Lamarck, 1816) & 1 & 1 & & 1 \\
\hline & Acropora palmata (Lamarck, 1816) & 1 & 1 & & 1 \\
\hline \multirow[t]{5}{*}{ Agariciidae } & Agaricia agaricites (Linnaeus, 1758) & 1 & 1 & & \\
\hline & Agaricia fragilis (Dana, 1846) & 1 & 1 & & \\
\hline & Agaricia humilis (Verril, 1901) & 1 & 1 & & \\
\hline & Agaricia lamarcki (Milne-Edwards \& Haime, 1846) & & 1 & & \\
\hline & Leptoseris cucullata (Ellis \& Solander, 1786) & 1 & 1 & & \\
\hline \multirow[t]{2}{*}{ Siderastreidae } & Siderastrea radians (Pallas, 1766) & 1 & 1 & 1 & 1 \\
\hline & Siderastrea siderea (Ellis \& Solander, 1786) & 1 & 1 & 1 & 1 \\
\hline \multirow[t]{5}{*}{ Poritidae } & Porites astreoides (Lamarck, 1816) & 1 & 1 & 1 & 1 \\
\hline & Porites branneri Rathbun, 1887 & 1 & & & \\
\hline & Porites colonensis (Zlatarski, 1990) & 1 & 1 & & \\
\hline & Porites porites $f$. furcata Lamarck, 1816 & & & & 1 \\
\hline & Porites porites $f$. porites (Pallas, 1766) & & & 1 & 1 \\
\hline Montastraeidae & Montastraea cavernosa (Linnaeus, 1758) & 1 & 1 & & 1 \\
\hline \multirow[t]{4}{*}{ Faviidae } & Colpophyllia natans (Houttuyn, 1772) & 1 & 1 & & 1 \\
\hline & Mycetophyllia lamarckiana (Milne-Edwards \& Haime, 1848) & 1 & 1 & & \\
\hline & Pseudodiploria clivosa (Ellis \& Solander, 1786) & 1 & & 1 & 1 \\
\hline & Pseudodiploria strigosa (Dana, 1846) & 1 & 1 & 1 & 1 \\
\hline \multirow[t]{3}{*}{ Merulinidae } & Orbicella annularis (Ellis \& Solander, 1786) & 1 & 1 & & 1 \\
\hline & Orbicella faveolata (Ellis \& Solander, 1786) & 1 & 1 & & \\
\hline & Orbicella franksi (Gregory, 1895) & 1 & 1 & & \\
\hline Rhizangiidae & Astrangia solitaria (Lesueur, 1817) & & 1 & & \\
\hline \multirow[t]{3}{*}{ Oculinidae } & Oculina diffusa (Lamarck, 1816) & 1 & 1 & & \\
\hline & Oculina robusta Pourtalès, 1871 & 1 & & & \\
\hline & Oculina varicosa (Lesueur, 1821) & 1 & 1 & & \\
\hline Meandrinidae & Dichocoenia stokesi Milne Edwards \& Haime, 1848 & & 1 & & \\
\hline \multirow[t]{3}{*}{ Mussidae } & Mussa angulosa (Pallas, 1766) & 1 & 1 & & \\
\hline & Scolymia cubensis (Milne Edwards \& Haime, 1849) & 1 & 1 & & \\
\hline & Scolymia lacera (Pallas, 1766) & & 1 & & \\
\hline \multirow[t]{2}{*}{ Caryophylliidae } & Paracyathus pulchellus (Philippi, 1842) & & 1 & & \\
\hline & Phyllangia americana Milne-Edwards y Haime, 1848 & & 1 & & \\
\hline Total & & 29 & 32 & 6 & 13 \\
\hline
\end{tabular}

de la pendiente de barlovento es parecida a la registrada para la cresta $(\mathrm{W}=1493,0, P=0,75)$. En las pendientes, la cobertura coralina es variable en función de los estratos de profundidad, pero mostró tendencias diferentes en barlovento y sotavento. En la primera, los valores más altos se ubicaron en el estrato de 10 a $20 \mathrm{~m}$ de profundidad $(11,3 \%$ en la capa de 10 a 15 m, $11,9 \%$ en la capa de 15 a
$20 \mathrm{~m}$ ) mientras que la cobertura menor se registró tanto en las áreas menores a $5 \mathrm{~m}$ como en las mayores a $25 \mathrm{~m}$ de profundidad (Fig. 4). El análisis de Kruskal-Wallis indicó diferencias significativas en la cobertura de barlovento $\left(\mathrm{H}_{(5,42)}=13,62, P=0,02\right)$. La prueba posterior mostró que las capas entre 10 y $20 \mathrm{~m}$ de profundidad, son diferentes del resto $(\mathrm{W}=537,0, P<0,05)$. 


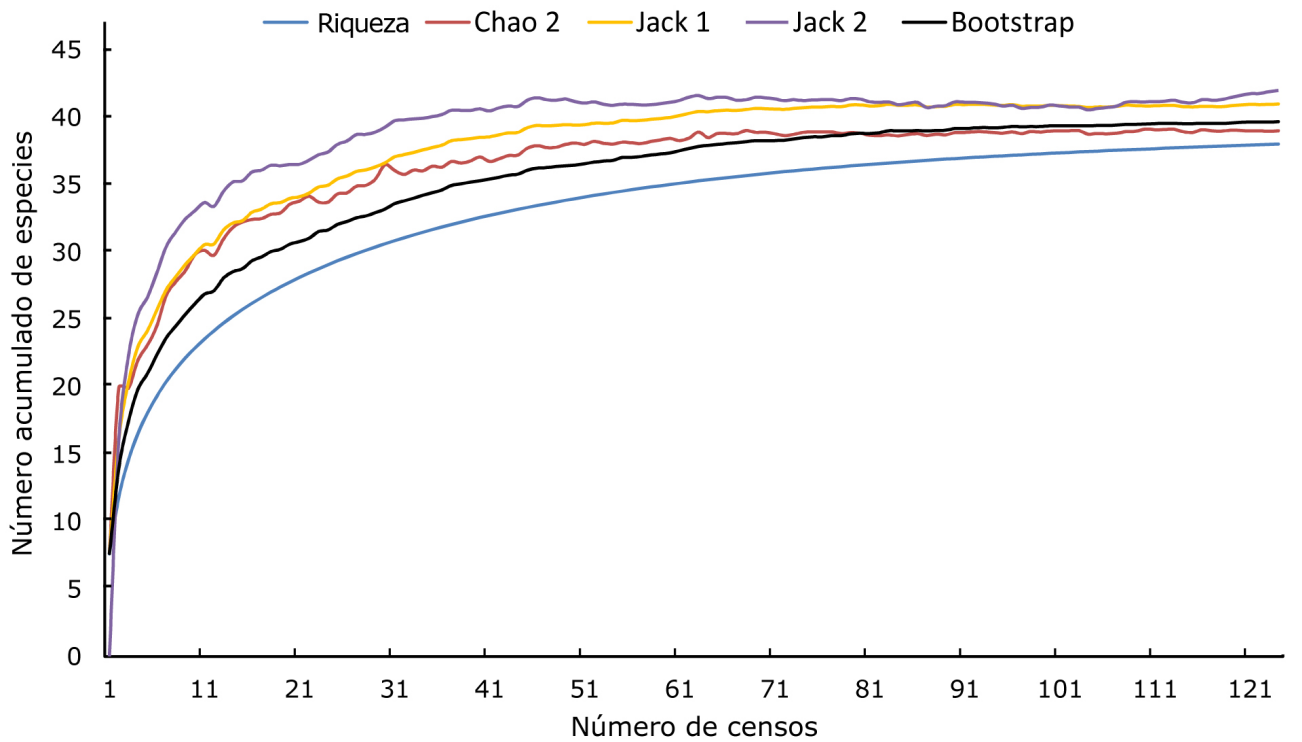

Figura 3. Curvas acumulativas de la riqueza coralina del arrecife Tuxpan, Veracruz, México, calculada con los modelos no paramétricos: Chao2, Jack1, Jack2 y Bootstrap / Cumulative curves of coral richness at Tuxpan reef, Veracruz, Mexico, calculated with no parametric models: Chao2, Jack1, Jack2 and Bootstrap

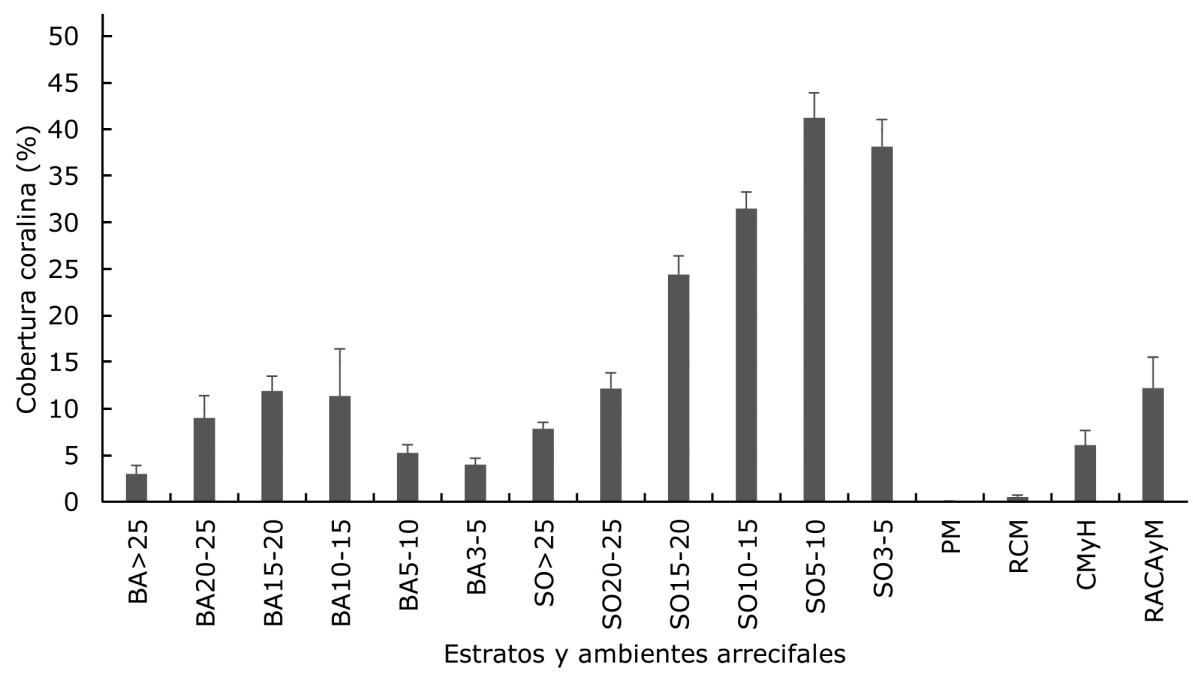

\begin{abstract}
Figura 4. Cobertura coralina por estrato de profundidad o ambiente del arrecife Tuxpan, Veracruz, México. BA= pendiente de barlovento; $\mathrm{SO}=$ pendiente de sotavento, $\mathrm{RACAyM}=$ roca con algas costrosas, Acropora $\mathrm{y}$ Millepora, $\mathrm{CMyH}=$ corales masivos $\mathrm{y}$ Halimeda, PM= pastos marinos, RCM= restos de coral muerto. 3-5, 5-10, 10-15, 15-20, 20-25 y >25 corresponden a los ámbitos de profundidad. Las barras corresponden a la media y las líneas al error estándar / Coral cover by depth layer or habitat of Tuxpan reef, Veracruz, Mexico. BA= windward slope; $\mathrm{SO}=$ leeward slope, $\mathrm{RACAyM}=$ rock with encrusting coralline algae, Acropora and Millepora, $\mathrm{CMyH}=$ massive corals and Halimeda, $\mathrm{PM}$ = seagrasses, $\mathrm{RCM}=$ fragments of dead coral. 3-5, 5-10, 10-15, 15-20, 20-25 and $>25$ correspond to depth layers. Bars correspond to mean and the lines to standard error
\end{abstract}

La cobertura coralina en la pendiente de sotavento mostró diferencias entre estratos de profundidad de acuerdo al $\operatorname{ANOVA}(\mathrm{F}(5,38)=18,73, P<0,05)$, y el análisis posterior, definió 3 grupos: $>20 \mathrm{~m}$, de 15 a $20 \mathrm{~m}$, y 3 a $15 \mathrm{~m} \mathrm{de}$ profundidad (Tabla 2). Por su parte, las zonas someras del arrecife (planicie y cresta arrecifal), revelaron valores bajos en las áreas de calma y más altos en las áreas con mayor dinámica (Fig. 4). La prueba de Kruskal-Wallis indica diferencias estadísticamente significativas $\left(\mathrm{H}_{(3,23)}=18,73\right.$, $P<0,05)$, donde las áreas de pastos marinos y restos de coral muerto, se separaron de las zonas de corales masivosHalimeda y Roca con algas costrosas, Acropora y Millepora $(\mathrm{W}=59,0, P<0,05)$. 
Tabla 2. Resultados de la prueba t-Student a los estratos de profundidad de la pendiente de sotavento del arrecife Tuxpan, Veracruz, México / Results of t-Student test to depth layers of leeward zone at Tuxpan Reef, Veracruz, Mexico

\begin{tabular}{lcc}
\hline Estratos comparados & $\mathrm{t}$ & $P$ \\
\hline$>20$ y $15-20 \mathrm{~m}$ & 5,03 & $<0,05$ \\
$>20$ y $3-15 \mathrm{~m}$ & 11,47 & $<0,05$ \\
$15-10$ y $3-15 \mathrm{~m}$ & 5,08 & $<0,05$ \\
\hline
\end{tabular}

Las especies de coral que más aportaron a la cobertura en el arrecife Tuxpan, en general, fueron las mismas para el sistema, pero sus proporciones fueron diferentes para cada zona arrecifal, en las pendientes fueron comunes: Colpophyllia natans, Siderastrea siderea y Orbicella franksi, en barlovento, además de las anteriores, dominaron: Acropora palmata y Montastraea cavernosa, en tanto que, en sotavento, $O$. annularis y $O$. faveolata contribuyeron de modo importante a la cobertura. En la cresta, las especies dominantes fueron: Pseudodiploria clivosa, M. alcicornis, Porites porites y A. palmata, en cambio en la laguna, dominaron Pseudodiploria strigosa, S. siderea y P. clivosa (Tabla 3).

Tabla 3. Porcentaje de cobertura de las especies coralinas por zona en el arrecife Tuxpan, Veracruz, México. En negritas se citan las 5 especies con mayor cobertura / Relative abundance of coral species by zone at Tuxpan reef, Veracruz, Mexico. In bold be show the 5 coral species with higher coverage

\begin{tabular}{lcccc}
\hline \multicolumn{1}{c}{ Especie } & Barlovento & Sotavento & Laguna & Cresta \\
\hline Acropora cervicornis & & 3,65 & & 3,55 \\
Acropora palmata & $\mathbf{1 4 , 0 7}$ & & & $\mathbf{9 , 1 0}$ \\
Colpophyllia natans & $\mathbf{1 7 , 0 6}$ & $\mathbf{2 2 , 4 3}$ & & 1,96 \\
Pseudodiploria clivosa & 5,78 & & $\mathbf{1 0 , 7 7}$ & $\mathbf{3 6 , 1 7}$ \\
Pseudodiploria strigosa & 6,91 & 2,77 & $\mathbf{3 5 , 8 4}$ & \\
Millepora alcicornis & 3,26 & & & $\mathbf{2 3 , 5 3}$ \\
Montastraea cavernosa & $\mathbf{1 1 , 2 7}$ & 7,37 & & 0,85 \\
Orbicella annularis & & $\mathbf{2 3 , 4 2}$ & & 1,73 \\
Orbicella faveolata & 9,29 & $\mathbf{1 0 , 8 0}$ & & \\
Orbicella franksi & $\mathbf{1 0 , 5 1}$ & $\mathbf{9 , 9 1}$ & & \\
Porites astreoides & & 1,09 & $\mathbf{3 , 3 4}$ & $\mathbf{7 , 9 0}$ \\
Porites porites & & & $\mathbf{2 0 , 6 8}$ & $\mathbf{9 , 2 8}$ \\
Porites furcata & & & & 5,08 \\
Siderastrea siderea & $\mathbf{1 1 , 3 5}$ & $\mathbf{9 , 9 8}$ & $\mathbf{2 9 , 3 7}$ & \\
Stephanocoenia intersepta & 2,59 & 4,76 & & 4,41 \\
Otras & 7,91 & 7,49 & & \\
\hline & & & & \\
\hline & & & & \\
& & & & \\
& & & & \\
& & & & \\
& & & & \\
\hline
\end{tabular}

A partir de la cobertura de los grupos morfo funcionales (GMF) y los corales escleractinios, el NMDS definió las zonas en el arrecife Tuxpan y esto es corroborado por el $\operatorname{ANOSIM}(\mathrm{R}=0,64, P<0,05)$. Las zonas arrecifales fueron: 1) la planicie arrecifal que reunió las áreas de pastos marinos y restos de coral muerto, caracterizadas por su limitada cobertura coralina $(<10 \%)$ y un alto porcentaje de material inerte, particularmente de arena y restos de coral muerto; 2) la cresta arrecifal, que incluyó áreas con rocas cubiertas de algas costrosas y los corales de los géneros: Acropora y Millepora; 3) la pendiente de barlovento, presentó corales ramificados y masivos; y 4) la pendiente de sotavento, con una cobertura mayor al $20 \%$ representada por las formas de tipo masiva, especialmente de los géneros Orbicella y Colpophyllia (Fig. 5, Tabla 4).

El análisis de correlación aplicado a la cobertura en la pendiente de sotavento, refiere que a medida que aumenta la profundidad, la cobertura coralina disminuye $(\mathrm{r}=-0,786$, $P<0,05$ ) y explica el $61,8 \%$ de la variabilidad (Fig. 6). En la pendiente de barlovento, no existe una relación con la profundidad $(\mathrm{r}=0,098, P=0,51)$.

Tabla 4. Resultados del ANOSIM calculados a partir de los grupos morfo funcionales y de cobertura coralina en el arrecife Tuxpan, Veracruz, México / ANOSIM results calculated from morpho functional groups and coral cover at Tuxpan Reef, Veracruz, Mexico

\begin{tabular}{lccc}
\hline \multicolumn{1}{c}{ Prueba entre grupos } & Estadístico R & $\begin{array}{c}\text { Nivel de } \\
\text { significancia (\%) }\end{array}$ & $\begin{array}{c}\text { Valor de } \\
P\end{array}$ \\
\hline Global & 0,643 & 0,01 & 0,0001 \\
Barlovento- Sotavento & 0,536 & 0,01 & 0,0001 \\
Barlovento- Planicie & 0,944 & 0,01 & 0,0001 \\
Barlovento- Cresta & 0,534 & 0,01 & 0,0001 \\
Sotavento-Planicie & 0,897 & 0,01 & 0,0001 \\
Sotavento-Cresta & 0,749 & 0,01 & 0,0001 \\
Planicie-Cresta & 0,825 & 0,01 & 0,0001 \\
\hline
\end{tabular}




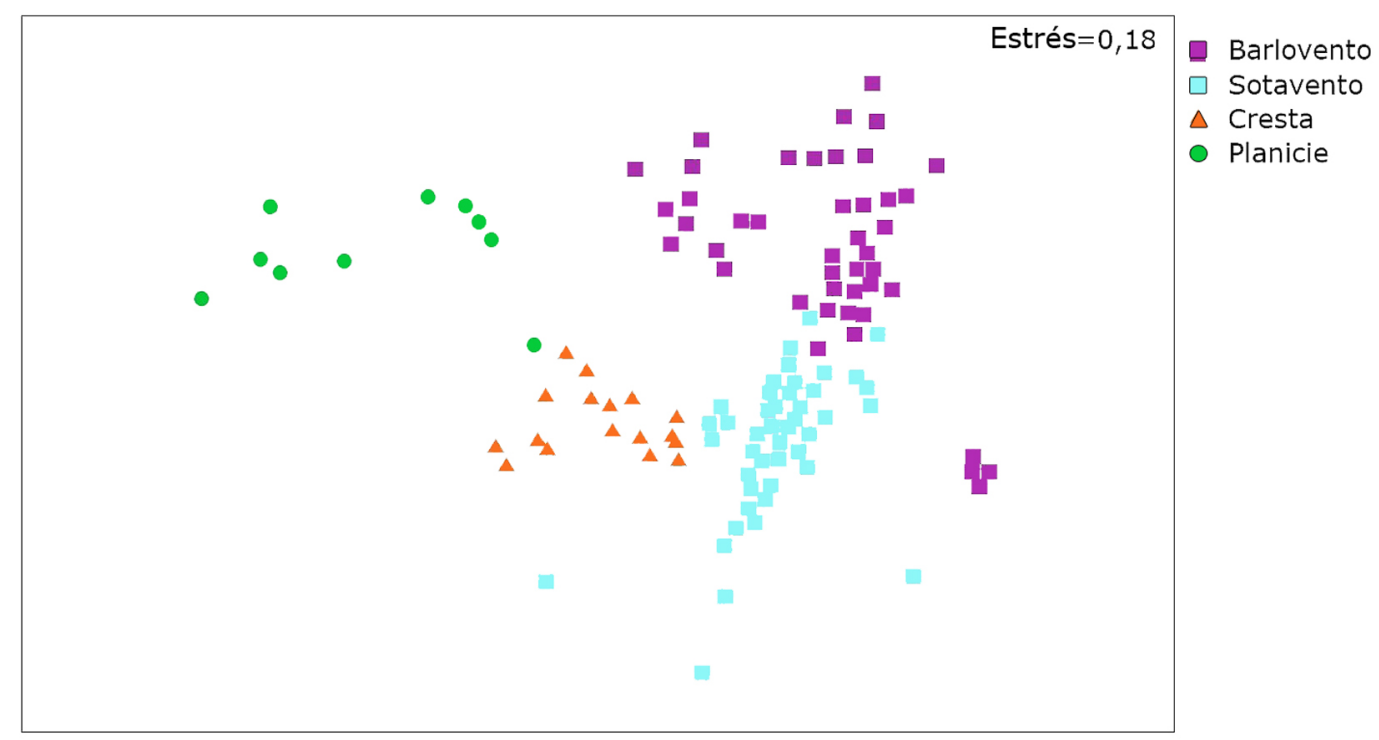

Figura 5. ANOSIM mostrando la zonación del arrecife Tuxpan, Veracruz, México a partir de los grupos morfo funcionales y de cobertura coralina / ANOSIM showing the zonation of Tuxpan reef, Veracruz, Mexico from the morpho functional groups and coral cover

\section{DisCUSIÓN}

La heterogeneidad del sustrato es un atributo típico de los sistemas arrecifales (Sheppard et al. 2009), los 7 grupos morfo funcionales que caracterizan al arrecife Tuxpan permiten definir las 4 zonas arrecifales (Fig. 5). La laguna o planicie arrecifal, que se identifica por la presencia de: pastos marinos, restos de coral muerto, arena y pequeñas colonias coralinas. En esta zona, la arena dominó en las áreas estabilizadas por los pastos marinos (Morgan \& Kitting 1984) y los corales que se presentaron en esta zona fueron: Porites porites y Pseudodiploria clivosa, mismas que han sido referidas previamente por Chávez et al. (1970) para la planicie del arrecife Lobos, en el SALT. La cresta arrecifal, presentó una alta proporción de algas verdes calcáreas (Halimeda opuntia) y algas rojas costrosas (Lithophyllum, Neogoliton y Porolithon), así como una cobertura coralina representada por especies propias de áreas con intenso oleaje, como es el caso de: Millepora, Porites y Acropora (Veron 2000, Jordan-Dahlgren \& Rodríguez Martínez 2003), parecido a lo registrado en otros sistemas de Veracruz (Horta-Puga et al. 2015) y del Banco de Campeche (Ferre D’Amare 1985, Carricart-Ganivet \& Beltrán-Torres 1997, Zarco-Perelló et al. 2013). La pendiente de barlovento, expuesta al oleaje y las corrientes superficiales definidas por los vientos del este en primavera-verano y del este-sureste en otoño invierno (Gutiérrez de Velazco \& Winant 1996), presenta una inclinación suave (Chávez et al. 2010, Alfaro 2012) con corales ramificados en las zonas menores a 10 $\mathrm{m}$ y masivos en las zonas entre 10 y $20 \mathrm{~m}$ de profundidad. La pendiente de sotavento que desciende abruptamente, protegida de los vientos dominantes (Anthony \& Larcombe 2000), cuyas aguas transparentes y de calma relativa favorecen el crecimiento y desarrollo coralino.

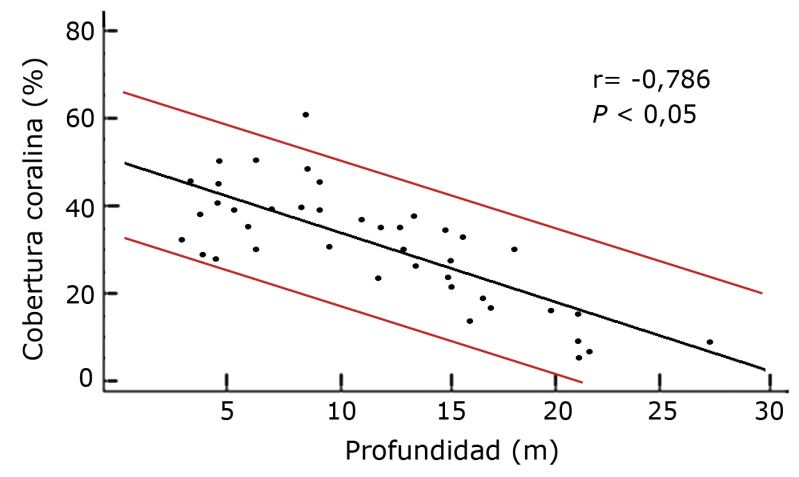

Figura 6. Relación de la cobertura coralina con la profundidad en la pendiente de sotavento del arrecife Tuxpan, Veracruz, México. Los puntos indican las muestras, la línea negra la ecuación de regresión y las líneas rojas los niveles de confianza / Relationship between coral cover and depth at leeward slope of Tuxpan reef, Veracruz, Mexico. Points indicate samples, black line, the regression equation and red lines the confidence levels 
El efecto del viento sobre la capa superficial del agua, en la pendiente de barlovento es más evidente en las capas menores a $10 \mathrm{~m}$ de profundidad, definiendo el establecimiento y desarrollo de algas costrosas (Chávez et al. 2010) y una limitada cobertura coralina. En contraste, las condiciones de transparencia de sotavento son mejores para el desarrollo coralino, particularmente en las áreas con menos de $20 \mathrm{~m}$ de profundidad. Sin embargo, la presencia de algas césped en esta zona es alta también (26,4\%), especialmente en las áreas cercanas al límite arrecifal, lo cual puede ser el resultado de la competencia por espacio con los corales (Swierts \&Vermeij 2016). Esto es debido a que los sedimentos finos propios del arrecife, se suspenden temporalmente (Larcombe et al. 1995) durante horas o días, cuando soplan los vientos del norte o del sur (Salas-Pérez et al. 2018), provocando la deposición sobre los corales, los cuales al morir favorecen el establecimiento de algas filamentosas (Nugues \& Roberts 2003). En la pendiente de sotavento la cobertura coralina es alta (Bayraktarov et al. 2014), porque sus aguas transparentes promueven el desarrollo coralino, particularmente de Orbicella spp., que actualmente, es el principal género constructor de arrecifes en Veracruz (Lara et al. 2002, Jordán-Dalhgren \& Rodríguez-Martínez 2003, Horta-Puga et al. 2015) y el Caribe (Jackson 1992, Bruckner \& Bruckner 2006, AlvarezFilip et al. 2011).

La riqueza coralina del arrecife Tuxpan (36 especies de corales escleractinios, un milleporino y un estilasterino (Tabla 1) fue similar a la registrada en el arrecife Lobos (Chávez et al. 1970, Escobar-Vázquez \& Chávez 2012) del SALT o los arrecifes Blanquilla (32 especies), Anegada de Afuera (31 especies) o Cabezo (30 especies) del SAV (Horta-Puga et al. 2015, Pérez-España et al. 2015), los cuales se conectan por las corrientes marinas (Salas-Monreal et al. 2018). La adición de nuevos registros para el arrecife Tuxpan es el reflejo del esfuerzo de investigación y de la exploración de ambientes no estudiados anteriormente (estratos de profundidad mayores a $20 \mathrm{~m}$ ). Por ejemplo, Agaricia lamarcki se observó únicamente en áreas de sotavento con más de $20 \mathrm{~m}$ de profundidad y Oculina robusta en la pendiente de barlovento a más de $25 \mathrm{~m}$. Además, los corales con talla menor a $1 \mathrm{~cm}$, como: Astrangia solitaria, Paracyathus pulchellus y Phillangia americana, pueden pasar desapercibidos o generar confusión, por lo que en estos casos, es menester recolectarlos para una revisión minuciosa al microscopio. La riqueza coralina es mayor en las pendientes (29 especies en barlovento y 32 en sotavento) con relación a la planicie (6 especies) y cresta arrecifal (12 especies), lo cual es producto de las condiciones particulares de cada zona y de la tolerancia de las especies a la luz, temperatura, profundidad e intensidad del oleaje entre otros (Jaap \& Hallock 1990, Bridge et al. 2013, Rogers et al. 2016).
La estructura de la comunidad coralina está influenciada por el ambiente oceánico donde se desarrolla el sistema (Lowe \& Falter 2015). Los ambientes lagunares del arrecife Tuxpan presentan una baja cobertura coralina: $0,1 \%$ en el área de restos de coral muerto y $0,5 \%$ en zona de pastos marinos y estuvo representada por: $P$. porites, $P$. clivosa, Pseudodiploria strigosa, Siderastrea radians y Porites astreoides, especies comunes de las planicies en los arrecifes de Veracruz (Lara et al. 1992, Carricart-Ganivet \& HortaPuga 1993, Martos 1993, Jordán-Dalhgren \& RodríguezMartínez 2003, Horta-Puga et al. 2015). Normalmente estos ambientes que están protegidos o alejados de la cresta son muy sensibles a los efectos del calentamiento local (Rogers et al.2016), debido a su limitada profundidad y por esto las especies que habitan en estas zonas deben ser muy tolerantes a esas fluctuaciones.

La cresta arrecifal es una zona de alta energía debido a su exposición al oleaje (Tunnell 1988). En el arrecife Tuxpan, la cobertura coralina en los ambientes de corales masivos y Halimeda $(6,1 \%)$ y Roca con algas costrosas, Acropora y Millepora (12,2\%) refleja la influencia del oleaje en sus componentes. Los corales Acropora palmata, Millepora alcicornis y $P$. clivosa, el alga calcárea $H$. opuntia y el gorgonáceo Erythropodium caribaeorum requieren de aguas transparentes, con buena circulación (Jaap \& Sargent 1993 ) y están adaptadas para soportar la energía del oleaje (Schuhmacher \& Plewka 1981).

La exposición al oleaje, las corrientes superficiales y el gradiente de exposición de la pendiente de barlovento pueden influir en la distribución de los corales escleractinios (Bayraktarov et al. 2014) y en su cobertura. De acuerdo con Rogers et al. (2016), las localidades con alto estrés del oleaje se asocian con una baja cobertura coralina, por esto, en el arrecife Tuxpan, esta cobertura es menor al 10\% en las zonas con menos de $10 \mathrm{~m}$ de profundidad. Además explica la presencia de especies adaptadas a esas condiciones de estrés (Lara et al. 1992, Jordan-Dahgren \& Rodríguez Martínez 2003, Chávez et al. 2010), como es el caso de: $A$. palmata, $P$. clivosa y $P$. strigosa. Los estudios efectuados por Escobar-Vázquez \& Chávez (2012) en el arrecife Lobos (SALT) y Horta-Puga et al. (2015) en arrecifes del SAV, refieren coberturas parecidas para barlovento, $11 \%$ en capas de 5 a 15 m en Lobos; 7,4\% en Anegada de Afuera, $8 \%$ en Cabezo y 9,2\% para En Medio en estratos de 5 a $10 \mathrm{~m}$. Sin embargo, la cobertura coralina para los arrecifes: Blanquilla (45,5\%), Sacrificios (20,3\%) y Pájaros $(34,0 \%)$ en barlovento es mayor. Estos contrastes parecen relacionarse con la exposición de los arrecifes al oleaje y las corrientes dominantes, los primeros están más expuestos al igual que el Tuxpan, en cambio los segundos están protegidos por una barrera artificial del puerto veracruzano. En las capas de barlovento con profundidad entre 10 y 25 $\mathrm{m}$, la cobertura oscila entre 10,5 y $12,3 \%$ y es dominada por 
corales masivos (Colpophyllia natans, Orbicella faveolata, Orbicella franksi y Siderastrea siderea) que soportan menos estrés del oleaje. Por otra parte, la menor cobertura en los estratos mayores a $25 \mathrm{~m}(3,0 \%)$ parece resultar de la menor penetración de luz y la cercanía al borde arrecifal, donde se genera suspensión de sedimentos y entre las especies dominantes están: Montastraea cavernosa y S. siderea.

La pendiente de sotavento presentó la cobertura coralina mayor $(30,2 \pm 1,9 \%)$, tendencia parecida a lo que se ha reportado para la mayoría de los arrecifes del SAV y de Lobos, en el SALT (Escobar-Vázquez \& Chávez 2012, Horta-Puga et al. 2015, Pérez España et al. 2015). El género Orbicella, es uno de los principales constructores arrecifales del Caribe y Golfo de México (Lara et al. 1992, Jordan-Dahgren \& Rodríguez Martínez 2003, Bruckner \& Bruckner 2006, Alvarez-Filip et al. 2011) y domina en esta zona del arrecife Tuxpan debido a la transparencia del agua y el escaso oleaje (Bayraktarov et al. 2014) que permiten la penetración de luz hasta $20 \mathrm{~m}$ o más de profundidad. Sin embargo, durante el verano los vientos temporales (horas a días) procedentes del sur y en el invierno los del norte (SalasPérez et al. 2018) producen la suspensión de sedimentos y su depósito en los tejidos coralinos que puede provocar su muerte y el desarrollo de poblaciones de algas césped que compiten espacialmente con los corales, especialmente durante el reclutamiento (Nugues \& Roberts 2003, Arnold et al. 2010, Ainswoth \& Mumby 2015, Swierts \& Vermeij 2016), aunque, las especies del género Orbicella son capaces de remover los sedimentos (Loya 1976).

Las especies dominantes en sotavento del arrecife Tuxpan (e.g., O. annularis, $C$. natans, $O$. faveolata, $S$. siderea y $O$. franksi) coinciden en general con las registradas en otras partes del Caribe (Bruckner \& Bruckner 2006, Pandolfi \& Jackson 2006, Alvarez-Filip et al. 2011, Bayraktarov et al. 2014) y el Golfo de México (Escobar-Vázquez \& Chávez 2012, Horta-Puga et al. 2015, Pérez España et al. 2015) a pesar de las condiciones de turbidez y sedimentos suspendidos (Tunnell 1988, 1992) que resultan del drenaje de las cuencas hidrológicas en las costas de Veracruz (Pérez-Maqueo et al. 2011). Las especies de los géneros: Montastraea, Mycetophyllia, Orbicella y Siderastrea están adaptados para sobrevivir a condiciones de estrés térmico, turbidez y sedimentos suspendidos entre otras, porque son eficientes removedores de sedimento (Loya 1976) o porque utilizan la materia orgánica suspendida como alimento (Lewis 1976). La suspensión temporal (horas o días) del sedimento en el arrecife Tuxpan (observación personal) fue más evidente en los límites del arrecife (capas mayores a $15 \mathrm{~m}$ ) donde la cobertura coralina es menor y las especies dominantes fueron: $C$. natans, $M$. cavernosa, $S$. siderea y Stephanocoenia intersepta.
El arrecife Tuxpan es uno de los más visitados por los turistas que practican esnórquel y buceo en el norte de Veracruz. De acuerdo con el plan de manejo del ANP, en este sistema, sólo se permiten actividades productivas de bajo impacto ambiental, que no impliquen modificaciones a las características naturales del arrecife (CONANP 2014). En la práctica, las actividades de esnórquel se permiten en la cresta y planicie donde habitan pequeñas colonias de corales frágiles (e.g., P. porites, A. palmata), mientras que el buceo se realiza en las zonas con profundidades entre 5 y $15 \mathrm{~m}$ (preferentemente en la pendiente de sotavento) donde la cobertura coralina es mayor al $20 \%$. Aunque los criterios de control de las autoridades son estrictos, es necesario fundamentarlos con información biológica, ya que el impacto continuo puede provocar una disminución en la cobertura y la diversidad de especies (Hernández et al. 2008). La resistencia de las comunidades coralinas al daño por turistas, se atribuye a las diferencias morfológicas de los corales y la estructura del sustrato (Hawkins \& Roberts 1992). Además, está documentado que la falta de información y de guías capacitados por parte de los prestadores de servicio repercute en el impacto sobre los organismos coralinos (Castro-Triana \& Pereira-Cháves 2016) que también depende de las habilidades y experiencia de los buzos (Krieger \& Chadwick 2013).

En la práctica de esnórquel en la planicie y cresta arrecifal del Tuxpan, se debe prohibir que los turistas pisen el fondo, porque en estas zonas hay colonias coralinas frágiles como: P. porites, A. palmata y M. alcicornis que se fragmentan con facilidad al contacto. Por otra parte, el buceo para principiantes debe ser regulado y permitido en la pendiente de barlovento a profundidades entre 5 y $10 \mathrm{~m}$, donde la cobertura coralina es baja $(<10 \%)$ y no hay sedimentos o bien en áreas arenosas de la pendiente de sotavento a una distancia mayor a $20 \mathrm{~m}$ del borde arrecifal. Durante el buceo, se debe poner atención especial a los buzos que toman fotografías, porque en el desarrollo de esta actividad pueden provocar daños al arrecife (Barker \& Roberts 2004) al tocar accidentalmente las colonias.

La información que se presenta en esta investigación genera un panorama más completo de la comunidad del bentos (grupos morfo funcionales y corales escleractinios) en arrecifes tipo plataforma de Veracruz, mostrando las diferencias en la riqueza coralina en las 4 zonas arrecifales así como los contrastes de la cobertura coralina en las pendientes. A partir de los GMF se caracterizan las 4 zonas del arrecife Tuxpan, lo cual puede ser muy útil para definir las áreas de recreación subacuática así como los mecanismos de control en los sistemas del ANP Lobos-Tuxpan. 


\section{Agradecimientos}

Esta investigación fue financiada por la Secretaría de Educación Pública, en el marco de la Red para el Análisis y Síntesis de la Zona Costera Veracruzana, Golfo de México y parcialmente por la CONABIO que financió el Proyecto "Esponjas, corales escleractinios, equinodermos y peces de los arrecifes coralinos del norte y sur de Veracruz (Convenio: FB1628/JF124/12). Se agradece el permiso de pesca de fomento emitido por la SAGARPA (DGOPA.5458.170512.1381).

\section{LITERATURA CITADA}

Ainswoth CH \& PJ Mumby. 2015. Coral-algal phase shifts alter fish communities and reduce fisheries production. Global Change Biology 21(1): 165-172. <doi:10.1111/gcb.12667>

Alfaro GKP. 2012. Modelo de distribución del hábitat bentónico para el arrecife Tuxpan, Veracruz. Tesis Licenciatura, Facultad de Ciencias Biológicas y Agropecuarias, Universidad Veracruzana, Tuxpan, 70 pp.

Alvarez-Filip L, IM Côte, JA Gill, AR Watkinson \& NK Dulvy. 2011. Region-wide temporal and spatial variation in Caribbean reef architecture: Is coral cover the whole story? Global Change Biology 17(7): 2470-2477.

Anthony KRN \& P Larcombe. 2000. Coral reefs in turbid waters: sediment-induced stresses in corals and likely mechanisms of adaptation. Proceedings $9^{\text {th }}$ International Coral Reef Symposium, Bali, 1: 239-244.

Arnold SN, RS Steneck \& PJ Mumby. 2010. Running the gauntlet: inhibitory effects of algal turfs on the process of coral recruitment. Marine Ecology Progress Series 414: 91-105.

Barker N \& C Roberts. 2004. Scuba diver behaviour and the management of diving impacts on coral reefs. Biological Conservation 120(4): 481-489.

Bayraktarov E, ML Bastidas-Salamanca \& C Wild. 2014. The physical environment in coral reefs of the Tayrona National Natural Park (Colombian Caribbean) in response to seasonal upwelling. Boletín de Investigaciones Marinas y Costeras 43(1): 137-157.

Bridge TC, TP Hughes, JM Guinotte \& P Bongaerts. 2013. Call to protect all coral reefs. Nature Climate Change 3: 528-530.

Bruckner AW \& RJ Bruckner. 2006. The recent decline of Montastraea annularis (complex) coral populations in western Curaçao: a cause for concern? Revista de Biología Tropical 54: 45-58.

Budd AF, H Fukami, ND Smith \& N Knowlton. 2012. Taxonomic classification of the reef coral family Mussidae (Cnidaria: Anthozoa: Scleractinia). Zoological Journal of the Linnean Society 166: 465-529.

Carricart-Ganivet JP \& AU Beltrán-Torres. 1997. Lista de corales pétreos (Hydrozoa: Milleporina, Stylasterina; Anthozoa: Scleractinia) de aguas someras del Banco de Campeche, México. Revista de Biología Tropical 44(3): 619-622.
Carricart-Ganivet JP \& G Horta-Puga. 1993. Arrecifes de Coral en México. En: Salazar-Vallejo SI \& NE González (eds). Biodiversidad marina y costera de México, pp. 80-90. CONABIO/CIQRO, México DF.

Castro-Triana LA \& JM Pereira-Cháves. 2016. Impacto de la actividad turística sobre los arrecifes coralinos del Parque Nacional Natural Corales del Rosario y San Bernardo, Colombia. Cuadernos de Investigación UNED 8(1): 17-26.

Chávez EA, E Hidalgo \& M Sevilla. 1970. Datos acerca de las comunidades bentónicas del arrecife Lobos, Veracruz. Revista de la Sociedad Mexicana de Historia Natural 31: 211-281.

Chávez EA, JW Tunnell Jr \& K Withers. 2010. Zonación y ecología de los arrecifes: Plataforma veracruzana y Banco de Campeche. En: Tunnell JW Jr, EA Chávez \& K Withers (eds). Arrecifes Coralinos del sur del Golfo de México, pp. 60-100. CICIMAR, La Paz.

Clarke KR. 1993. Non-parametric multivariate analyses of changes in community structure. Australian Journal of Ecology 18: 117-143.

Clarke KR \& RN Gorley. 2006. PERMANOVA+ for PRIMER: Guide to Software and Statistical Methods. 214 pp. PRIMER-E, Plymouth.

Colwell RK. 2013. EstimateS: Statistical estimation of species richness and shared species from samples. Version 9. < purl. oclc.org/estimates $>$.

CONANP. 2014. Programa de Manejo Área de Protección de Flora y Fauna Sistema Arrecifal Lobos-Tuxpan, 175 pp. Comisión Nacional de Áreas Naturales Protegidas, Secretaría de Medio Ambiente y Recursos Naturales, México.

Erftemeijer PL, AB Rieglc, BW Hoeksemad \& PA Todde. 2012. Environmental impacts of dredging and other sediment disturbances on corals: A review. Marine Pollution Bulletin 64: 1737-1765.

Escobar-Vázquez C \& E Chávez. 2012. Coral community structure at Isla Lobos reef, Gulf of México. Biodiversity and systematic. In: Proceedings of the 12th International Coral Reef Symposium, Cairns, pp. 1-4.

Fabricious KE, M Logan, S Weeks \& J Brodie. 2014. The effects of river run-off on water clarity across the central Great Barrier Reef. Marine Pollution Bulletin 84: 191-200.

Ferre-D'Amare AR. 1985. Coral reefs of the Mexican Atlantic: A review. In: Proceedings of the 5th International Coral Reef Symposium, Tahití, 6: 349-354.

Gutiérrez de Velazco G \& C Winant. 1996. Seasonal patterns of wind stress and wind stress curl over the Gulf of Mexico. Journal of Geophysical Research 101: 18127-18140.

Hammer O, DAT Harper \& PD Ryan. 2001. PAST: Palaentological statistics software package for education and data analysis. Palaeontologia Electronica 4(1), 1-9. $<$ https:// palaeo-electronica.org/2001_1/past/past.pdf>

Hawkins JP \& CM Roberts. 1992. Effects of recreational SCUBA diving on fore-reef slope communities of coral reefs. Biological Conservation 62: 171-178. 
Hernández L, L Rodríguez, K Monticone \& E De la Guardia. 2008. Incidencias del buceo recreativo sobre los arrecifes coralinos en Cayo Coco, Cuba. Revista de Investigaciones Marinas 29(3): 205-212.

Horta-Puga G, JL Tello-Musi, A Beltrán-Torres, JP Carricart-Ganivet, JD Carriquiry \& J VillaescusaCelaya. 2015. Veracruz reef system: a hermatypic coral community thriving in a sedimentary terrigenous environment. En: Granados-Barba A, LD Ortiz-Lozano, D Salas-Monreal \& C González-Gándara (eds). Aportes al conocimiento del Sistema Arrecifal Veracruzano. Hacia el corredor arrecifal del suroeste del Golfo de México, pp. 181-207. Universidad Autónoma de Campeche, Campeche.

Humann P \& N Deloach. 2013. Reef coral identification Florida Caribbean Bahamas, 270 pp. New World Publications, Jacksonville.

Jaap WC \& P Hallock. 1990. Coral reefs. In: Myers RL \& JJ Ewel (eds). Ecosystems of Florida, pp. 574-616. University Press of Florida, Gainesville.

Jaap WC \& FJ Sargent. 1993. The status of the remnant populations of Acropora palmata (Lamarck, 1816) at Dry Tortugas National Park, Florida, with a discussion of possible causes of changes since 1881. In: Ginsburg RN (ed). Global aspects of coral reefs, pp. 101-104. University of Miami, Miami.

Jackson JBC. 1992. Pleistocene perspectives on coral reef community structure. American Zoologist 32: 719-731.

Jordan-Dahlgren E \& RE Rodríguez-Martínez. 2003. The Atlantic coral reefs of México. In: Cortes J (ed). Latin American coral reefs, pp. 131-158. Elsevier Science, Amsterdam.

Kohler KE \& SM Gill. 2006. Coral Point Count with Excel extensions (CPCe): A Visual Basic program for the determination of coral and substrate coverage using random point count methodology. Computers and Geosiences 32(9): 1259-1269.

Krieger JR \& NE Chadwick. 2013. Recreational diving impacts and the use of pre-dive briefings as a management strategy on Florida coral reefs. Journal of Coastal Conservation 17(1): 179-189.

Lang JC, KW Marks, PA Kramer, PR Kramer \& RN Ginsburg. 2015. Agrra Protocols Version 5.5, 44 pp. Atlantic and Gulf Rapid Reef Assessment, AGRRA < http://www. healthyreefs.org/cms/wp-content/uploads/2013/07/Protoc.AGRRA-V5.5-FINAL-Espanol.pdf>

Lara M, C Padilla, C García \& JJ Espejel. 1992. Coral reefs of Veracruz, Mexico, I: Zonation and community. In: Proceedings of the 7th International Coral Reef Symposium, Mangilao, Guam 1: 535-544.

Larcombe P, PV Ridd, A Prytz \& B Wilson. 1995. Factors controlling suspended sediment on the inner-shelf coral reefs, Townsville, Australia. Coral Reefs 14: 163-171.

Lewis JB. 1976. Experimental tests of suspension feeding in Atlantic reef corals. Marine Biology 36(2): 147-150.

Lowe RJ \& JL Falter. 2015. Oceanic forcing of coral reefs. Annual Review of Marine Science 7: 43-66.

Loya Y. 1976. Recolonization of Red Sea corals affected by natural catastrophes and man-made perturbations. Ecology 57: $278-289$.
Martos FFJ. 1993. Estudio sobre corales escleractinios (Cnidaria:Anthozoa, Scleractinia) del arrecife Tuxpan, Veracruz. Tesis Licenciatura, Facultad de Biología, Universidad Veracruzana, Tuxpan, 54 pp.

Morgan MD \& CI Kitting. 1984. Productivity and utilization of the seagrass Halodule wrightii and its attached epiphytes. Limnology and Oceanography 29: 1066-1076.

Muthiga NA \& TR McClanahan. 1997. The effect of visitor use on the bard coral communities of the Kisite Marine Park, Kenya. Proceedings of 8th International Coral Reef Symposium 2: 1879-1882.

Nugues MM \& CM Roberts. 2003. Coral mortality and interaction with algae in relation to sedimentation. Coral Reefs 22: 507-516.

Ortiz-Lozano LD, H Pérez-España, A Granados-Barba, C González-Gándara, A Gutiérrez-Velázquez \& J Martos. 2013. The Reef Corridor of the Southwest Gulf of Mexico: Challenges for its management and conservation. Ocean and Coastal Management 86: 22-32.

Pandolfi JM \& JBC Jackson. 2006. Ecological persistence interrupted in Caribbean coral reefs. Ecology Letters 9: 818-826.

Pérez-España H, S Ávila-Gutiérrez, SM Melo-Merino, P Berumen-Solórzano \& RR Flores-Arévalo. 2015. Patrones interanuales e interarrecifales de las comunidades de peces, corales y equinodermos en el Sistema Arrecifal Veracruzano. En: Granados-Barba A, LD Ortiz-Lozano, D Salas-Monreal \& C González-Gándara (eds). Aportes al conocimiento del Sistema Arrecifal Veracruzano. Hacia el corredor arrecifal del suroeste del Golfo de México, pp. 159-178. Universidad Autónoma de Campeche, Campeche.

Pérez-Maqueo O, L Muñoz-Villiers, G Vázquez, EZ Equihua \& RP León. 2011. Hidrología. En: Cruz-Angón A (ed). La biodiversidad en Veracruz estudio de estado1: 289-292. CONABIO, Gobierno de Veracruz, Universidad Veracruzana, Instituto de Ecología, Xalapa.

Rogers JS, SG Monismith, DA Koweek, WI Torres \& RB Dunbar. 2016. Thermodynamics and hydrodynamics in the atoll reef system and their influence on coral cover. Limnology and Oceanography 61: 2191-2206.

Salas-Monreal D, M Marín-Hernández, JJ Salas-Pérez, DA Salas-de-León, MA Monreal-Gómez \& H Pérez-España. 2018. Coral reef connectivity within the Wester Gulf of Mexico. Journal of Marine Systems 170: 88-99.

Salas-Pérez JJ, AG Jordán-Garza, D Salas-Monreal, C González-Gándara \& C Domínguez-Barradas. 2018. Features and circulation of water masses in the reef corridor of the southwestern Gulf of Mexico. In: Salas-Pérez JJ \& AG Jordan-Garza (eds). Oceanography of the Reef Corridor of the Southwestern Gulf of Mexico, pp. 39-66. Nova Science Publisher, New York.

Schuhmacher H \& M Plewka. 1981. The adaptive significance of mechanical properties versus morphological adjustments in skeletons of Acropora palmata and Acropora cervicornis (Cnidaria, Scleractinia). In: Gomez ED, CE Birkeland, RW Buddemeier, RE Johannes, JA Marsh Jr \& RT Tsuda (eds). Proceedings of 4th International Coral Reef Symposium, Vol. 2. Marine Science Center, University of the Philippines, Manila, pp. 121-128. 
Sheppard CRC, SK Davy \& GM Pilling. 2009. The biology of coral reefs, 339 pp. Oxford University Press, Oxford.

Swierts T \& MJA Vermeij. 2016. Competitive interactions between corals and turf algae depend on coral colony form. PeerJ 4, < doi:10.7717/peerj.1984>

Tunnell JW Jr. 1988. Regional comparison of southwestern Gulf of Mexico to Caribbean Sea coral reefs. In: Proceedings of the 6th International Coral Reef Symposium 3: 303-308.

Tunnell JW Jr. 1992. Natural versus human impacts to Southern Gulf of Mexico coral reef resources. In: Proceedings of the 7th International Coral Reef Symposium 1: 300-305.

Veron J. 2000. Corals of the world, Volumes 1-3: 1-1410. Australian Institute of Marine Sciences, Melbourne.

Wenger AS, DH Williamson, ET da Silva, DM Ceccarelli, NK Browne, C Petus \& MJ Devlin. 2015. Effects of reduced water quality on coral reefs in and out of no-take marine reserves. Conservation Biology 30(1): 142-153. < doi: 10.1111/cobi.12576>
WoRMS. 2019. World Register of Marine Species. WoRMS Editorial Board, <http://www.marinespecies.org > at VLIZ. $<$ doi:10.14284/170>

Zarco-Perelló S, M Mascaró, R Garza-Pérez \& N Simoes. 2013. Topography and coral community of the Sisal Reefs, Campeche Bank, Yucatán, México. Hidrobiológica 23(1): 28-41.

Zlatarsky VN \& N Martínez-Estalella. 2018. Los escleractinios de Cuba con datos sobre sus organismos asociados, $471 \mathrm{pp}$. Harte Research Institute for Gulf of Mexico Studies at Texas A\&M University, Corpus Christi.

Recibido el 14 de mayo 2019 y aceptado el 13 de agosto de 2019

Editor: Claudia Bustos D. 